\title{
Profiling Supercooled Liquid Water Clouds with Multi-Frequency Radar
}

Ian S. Adams, S. Joseph Munchak, Lihua Li, Paul Racette, Dong L. Wu, Gerald M. Heymsfield, and Adrian Loftus*

NASA Goddard Space Flight Center (GSFC)

*also with Earth System Science Interdisciplinary Center,

University of Maryland (ESSIC, UMd)

Special Thanks to Rachael Kroodsma (GSFC \& ESSIC, UMd),

Kuo-Sen Kuo (GSFC \& ESSIC, UMd), Craig Pelissier (GSFC \& SSAI), Thomas Clune (GSFC) 


\section{Motivation}

Mixed-phase clouds are an important variable in the Earth system

- Important component in microphysical processes

- Key parameter in climate radiation budget

- Impactful condition in aviation safety

Quantification of mixed-phase clouds on wide scales lacking

- Ground-based radar/lidar combinations provide some information

o Observations are localized, e.g., Barrow, Alaska

o Droplets typically at or below radar detectability limits

o Lidar returns rapidly extinguished by liquid 


\section{Approach}

Exploit differential measurements

- Backscatter (Rayleigh vs. non)

- Extinction (mainly gasses, cloud)

Multiparameter estimation

- Mass-weighted mean size $\left(\mathrm{D}_{\mathrm{m}}\right)$

- Precipitation water content

- Cloud water content

- Pristine / aggregate fraction

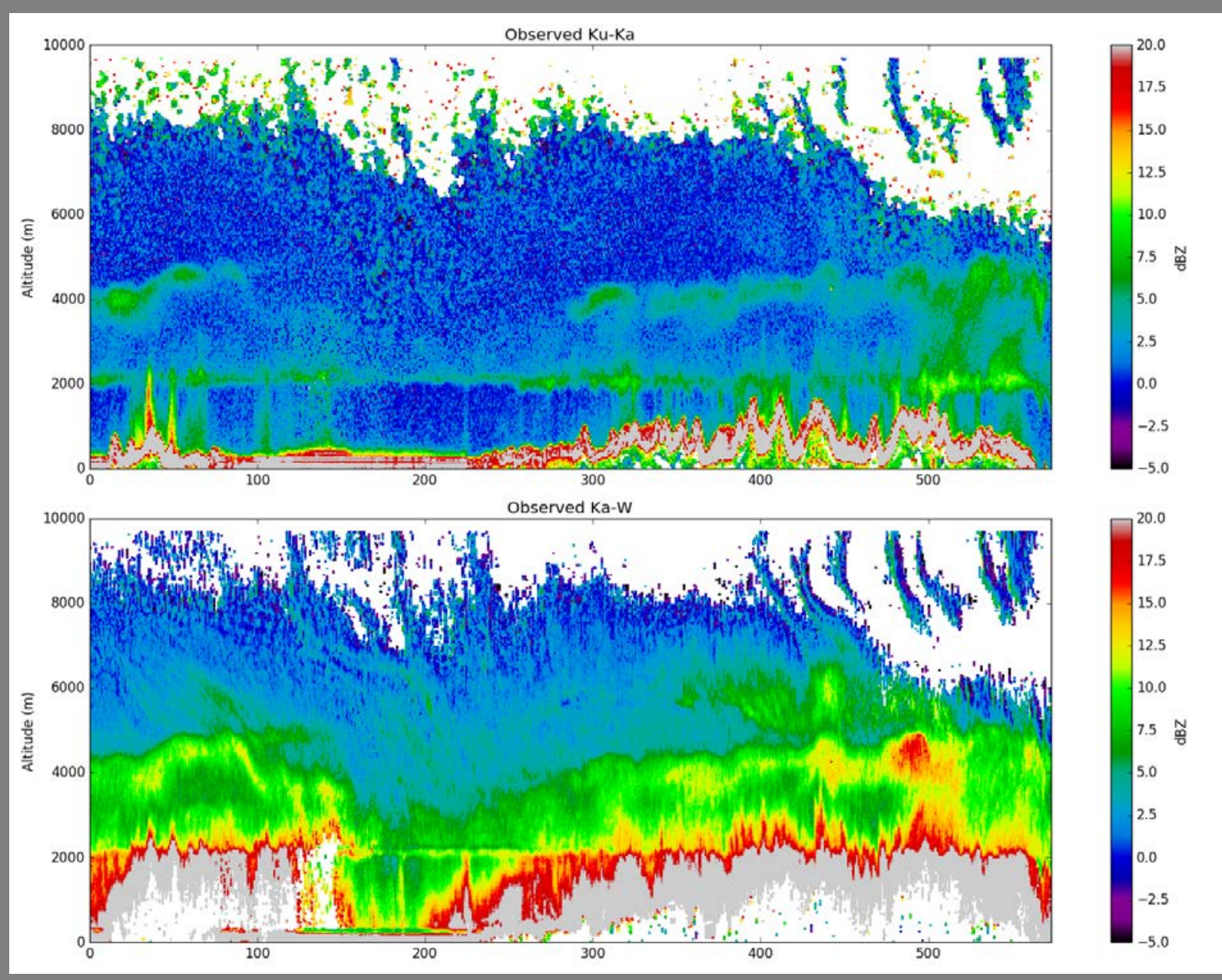




\section{Triple frequency space}

- Particle density

- Extinction

\section{Consistent forward modeling}

- Scattering tables

- Rosenkranz gas absorption

o Move to HITRAN + MT_CKD (AER)

- Liquid Permittivity (Turner et al. 2016)

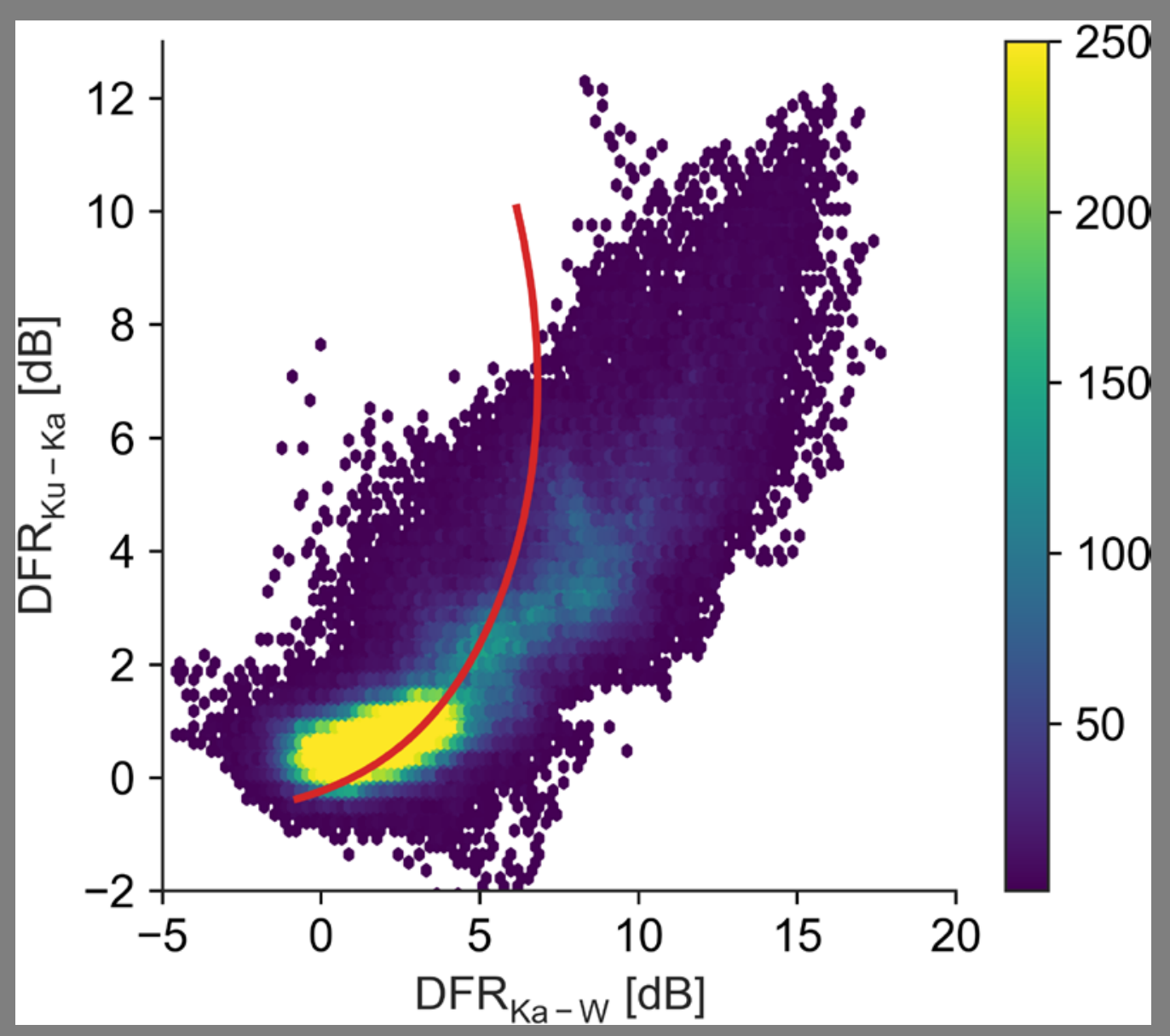

https://storm.pps.eosdis.nasa.gov/storm/OpenSSP.jsp 


\section{Scattering Tables}

https://storm. pps.eosdis. nasa.gov/storm/OpenSSP.jsp

Consistent hydrometeor scattering tables are necessary for consistent forward modeling of multifrequency observations

- Depositional growth model

o Reproduces planar and columnar geometries found in nature

- Aggregation performed heuristically

o Randomly oriented

- Horizontally-oriented plates

o T-matrix
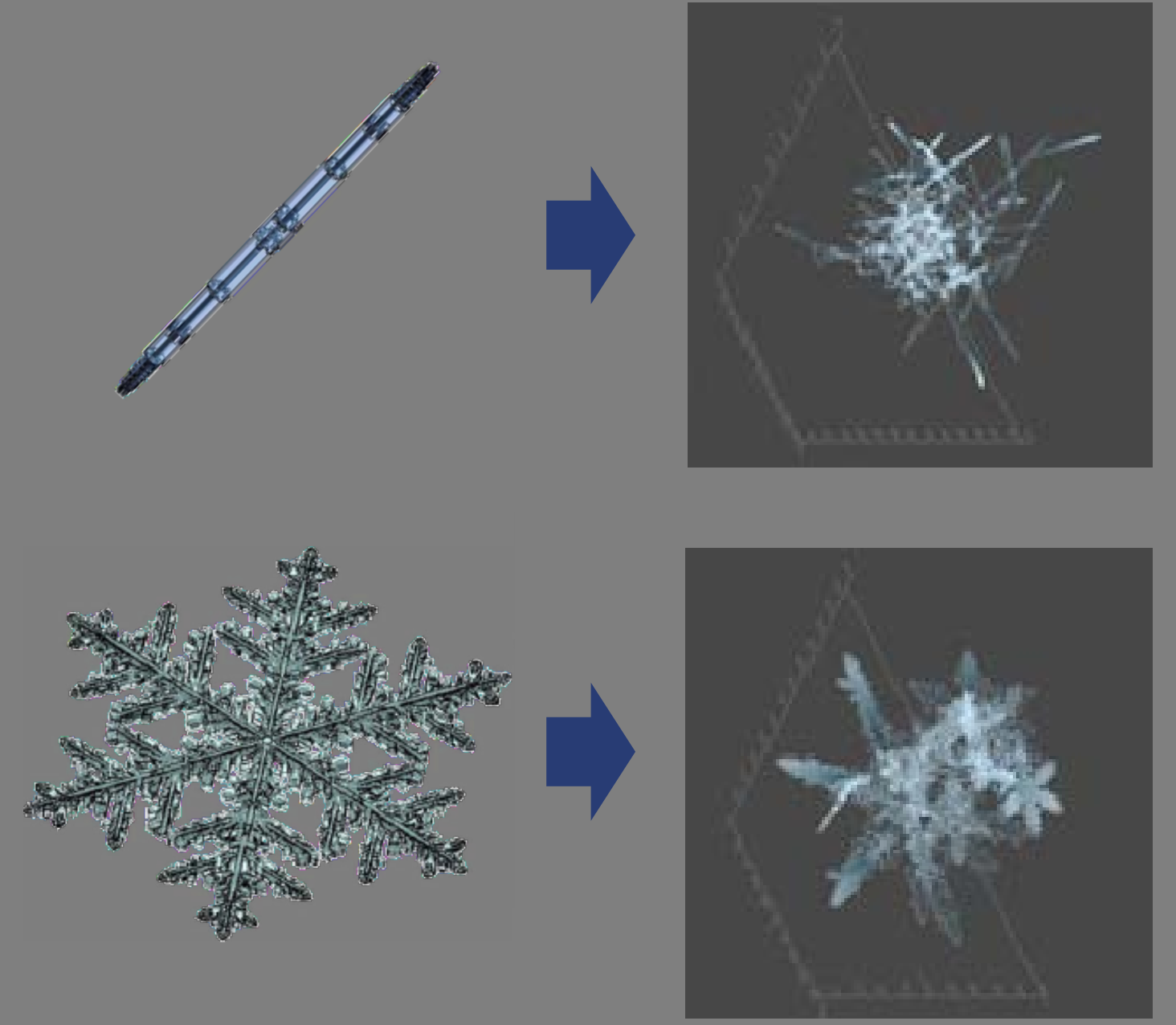


\section{Olympic Mountains Experiment (OLYMPEX)}

\begin{tabular}{|c|c|c|}
\hline DC-8 & Citation & ER-2 (Radar Definition Experiment) \\
\hline \multirow{3}{*}{$\begin{array}{l}\text { CoSMIR } \\
50,89,165,183+/-1,3,8 \mathrm{GHz} \\
\text { Conical and cross track scans } \\
\text { Fixed polarization basis }\end{array}$} & King Hot Wire Probe LWC & $\begin{array}{l}\text { AMPR } \\
10.7,19.35,37.1,85.5 \mathrm{GHz}\end{array}$ \\
\hline & CDP Cloud droplet size distribution & $\begin{array}{l}\text { HIWRAP } \\
\text { Ku, Ka bands; Nadir pointing }\end{array}$ \\
\hline & 2D-S Particle images & \multirow{2}{*}{$\begin{array}{l}\text { CRS } \\
\text { W band; Nadir pointing }\end{array}$} \\
\hline \multirow{3}{*}{$\begin{array}{l}\text { APR-3 } \\
\text { Ku, Ka, W band (dual polarization) } \\
\text { Cross-track scan }\end{array}$} & HVPS-3 (x2) Particle images & \\
\hline & Cloud Particle Imager (CPI) & $\begin{array}{l}\text { EXRAD } \\
\text { X band; Nadir pointing; Conical scan }\end{array}$ \\
\hline & CSI Cloud water content & $\begin{array}{l}\text { AirMSPI } \\
8 \text { bands (355-935 nm) }\end{array}$ \\
\hline Pressure & 2DC Particle images & CPL \\
\hline Temperature & Nevzorov Total water content & $355,532,1064 \mathrm{~nm}$ \\
\hline $\begin{array}{l}\text { Relative humidity } \\
\text { Wind }\end{array}$ & Rosemonunt Icing Probe & $\begin{array}{l}\text { eMAS } \\
38 \text { bands }(0.4-15 \mu \mathrm{m})\end{array}$ \\
\hline
\end{tabular}




\section{OLYPEX Case Study}

\section{December 2015}

- DC-8 and ER-2 flights

o Focus on APR-3 (DC-8)

- Citation

o Stacked microphysics legs

o Qualitative comparisons

o Range of frozen habits

o Presence of supercooled liquid clouds

APR-3

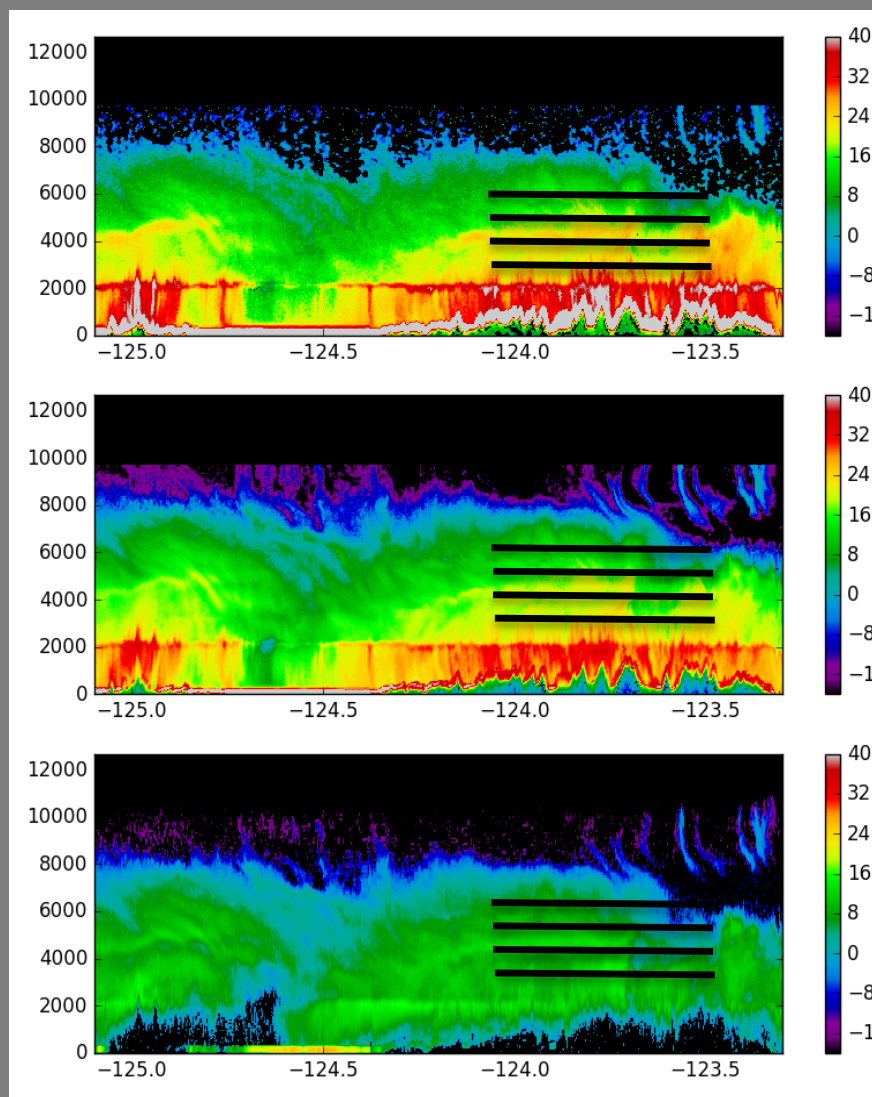

$-124.0$
COSMIR

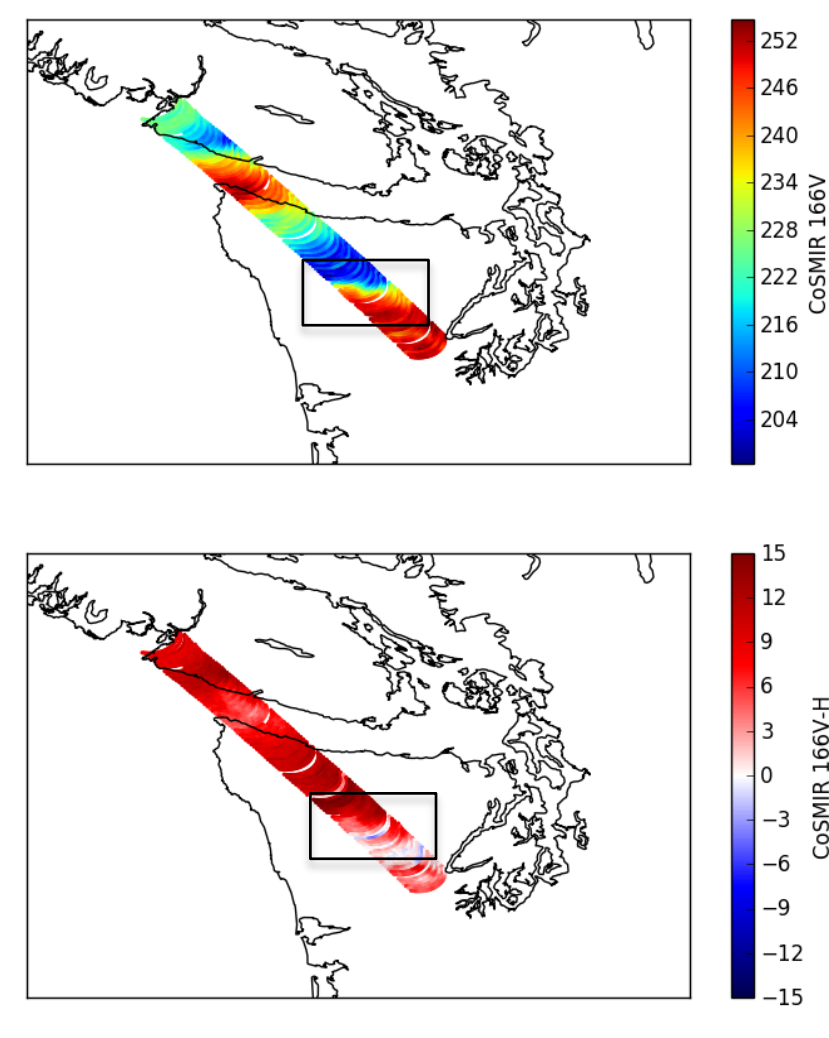




\section{Baseline Microphysics}

\section{Hitschfeld-Bordan} retrieval (1954)

- Estimate $D_{m}$

o Temperature dependent

- Default $N_{w}$ profile o Depends on $\mathrm{D}_{\mathrm{m}}$

- 50\% aggregate / pristine mix

- Mean profiles

o Temperature

- Water vapor

o Cloud liquid water

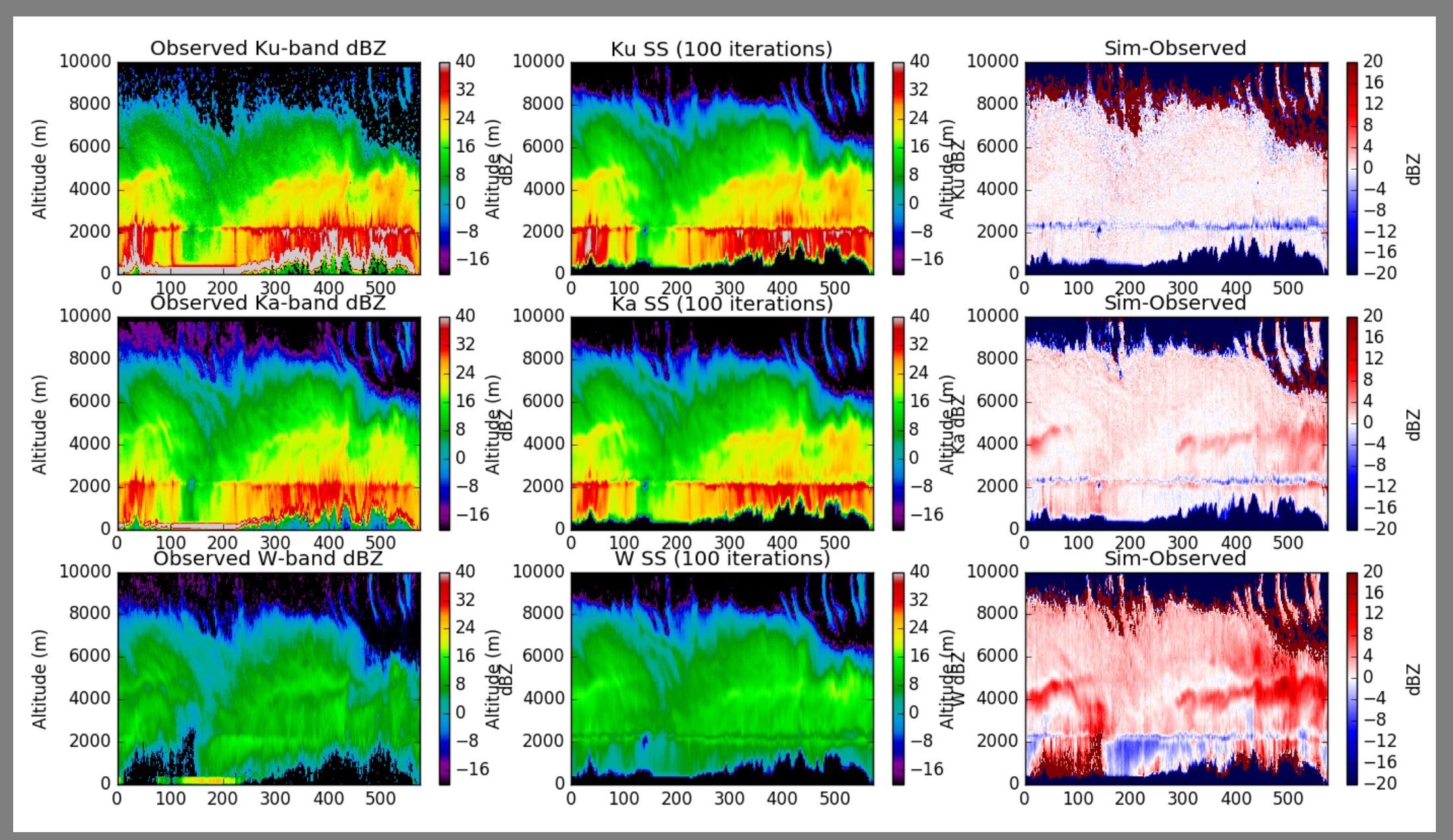


- Retrievals match probes o Good qualitative match

- Bands of increased reflectivity correspond to large $D_{m}$ and high aggregate fraction

- Significant amounts of supercooled liquid water
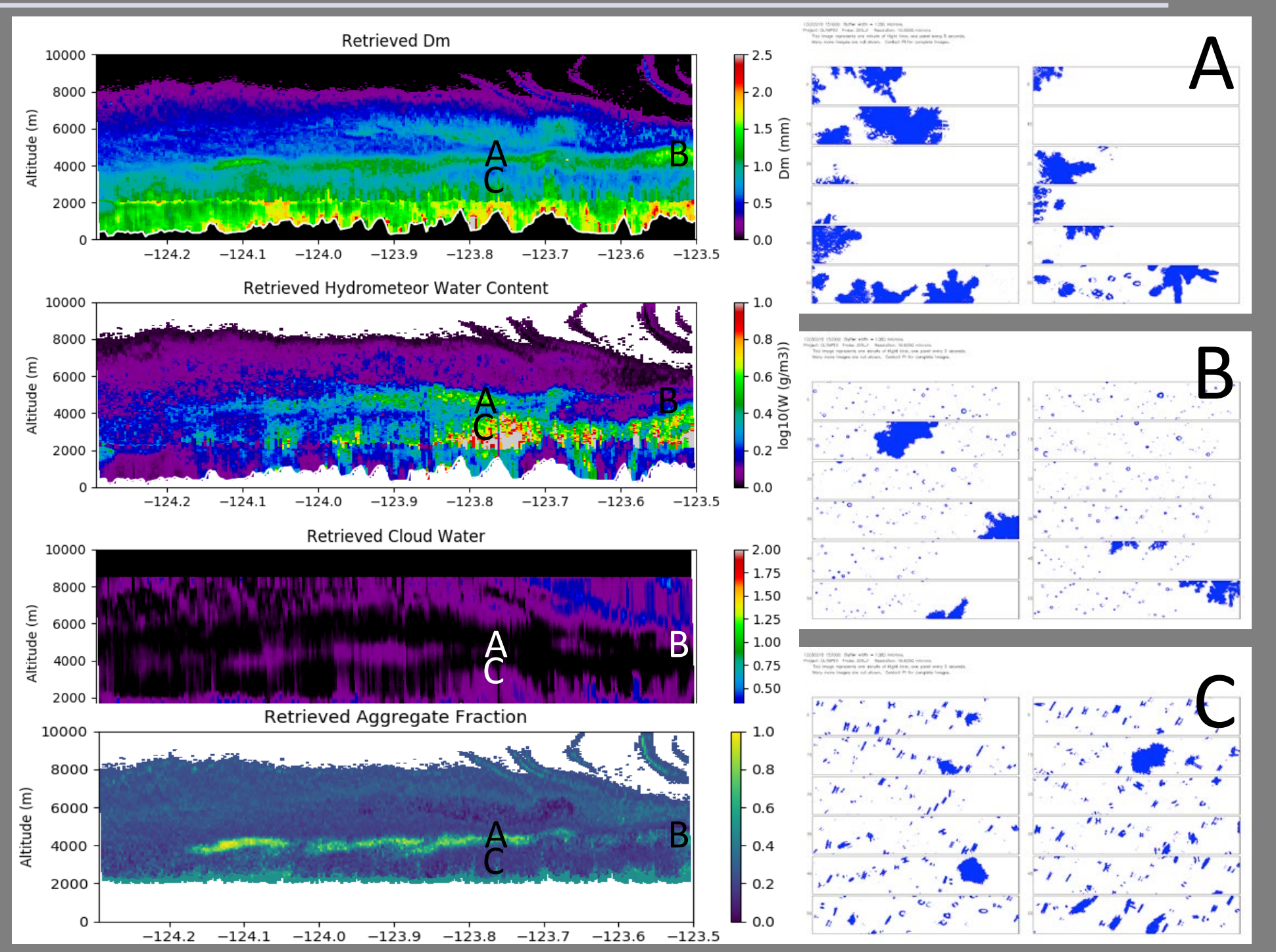


\section{Beyond 1D Radar Retrievals}

Three-dimensional effects not usually an issue for narrow radar beams; multiple scattering enhancement apparent at W-band

- Spatially dependent phenomenon Additional information in polarimetric observations

- Particle alignment

- Hydrometeor discrimination

- Melting layer

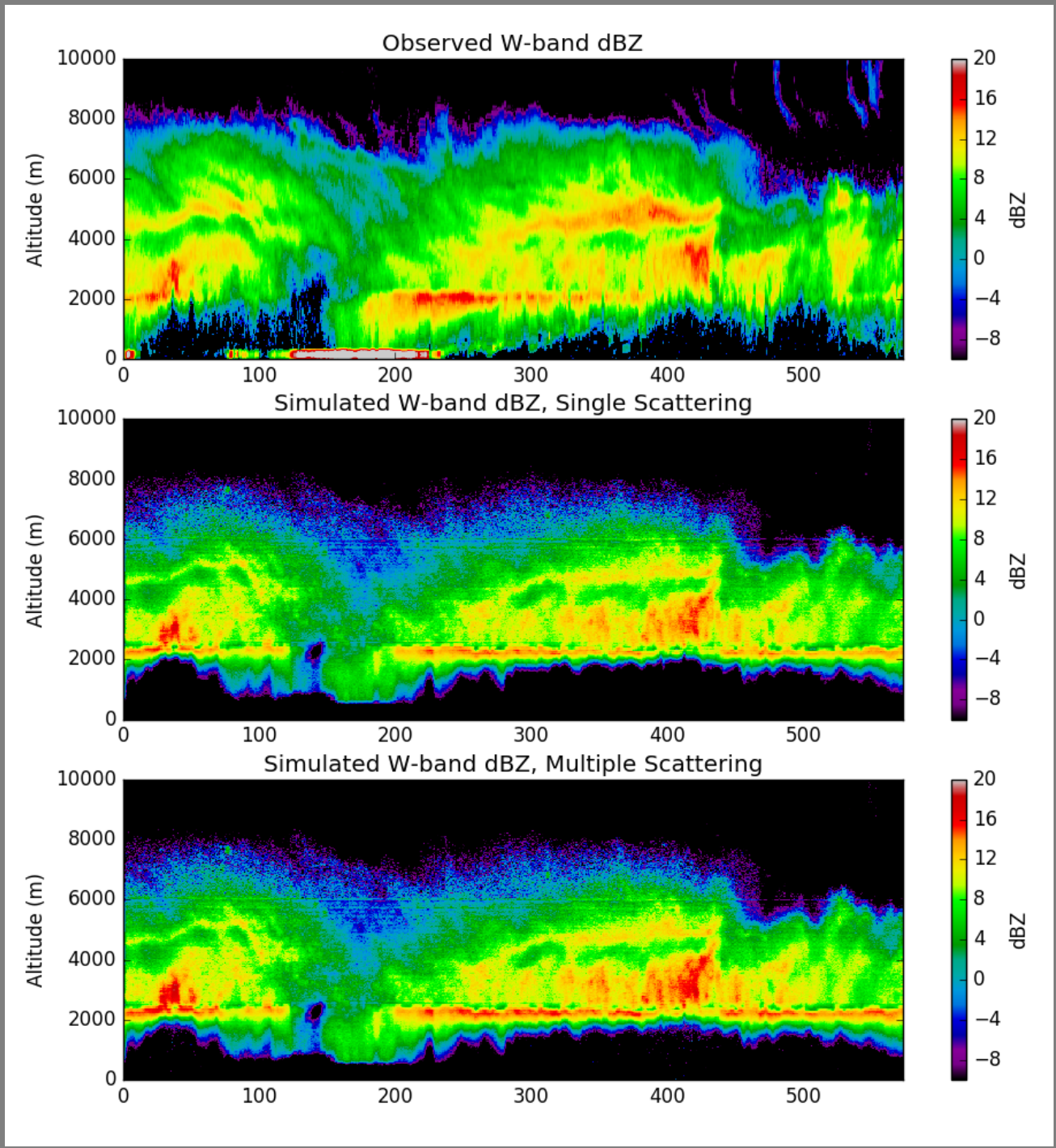




\section{Radiometers}

\section{Polarized mmwave brightness}

\section{temperatures provide additional} information on clouds and snow

- Aligned oblate/prolate particles

- Randomly oriented or small aspect ratio

- Differentiation between stratiform and deep convection

- Damping of polarization due to liquid water clouds (Panegrossi et al. 2017)

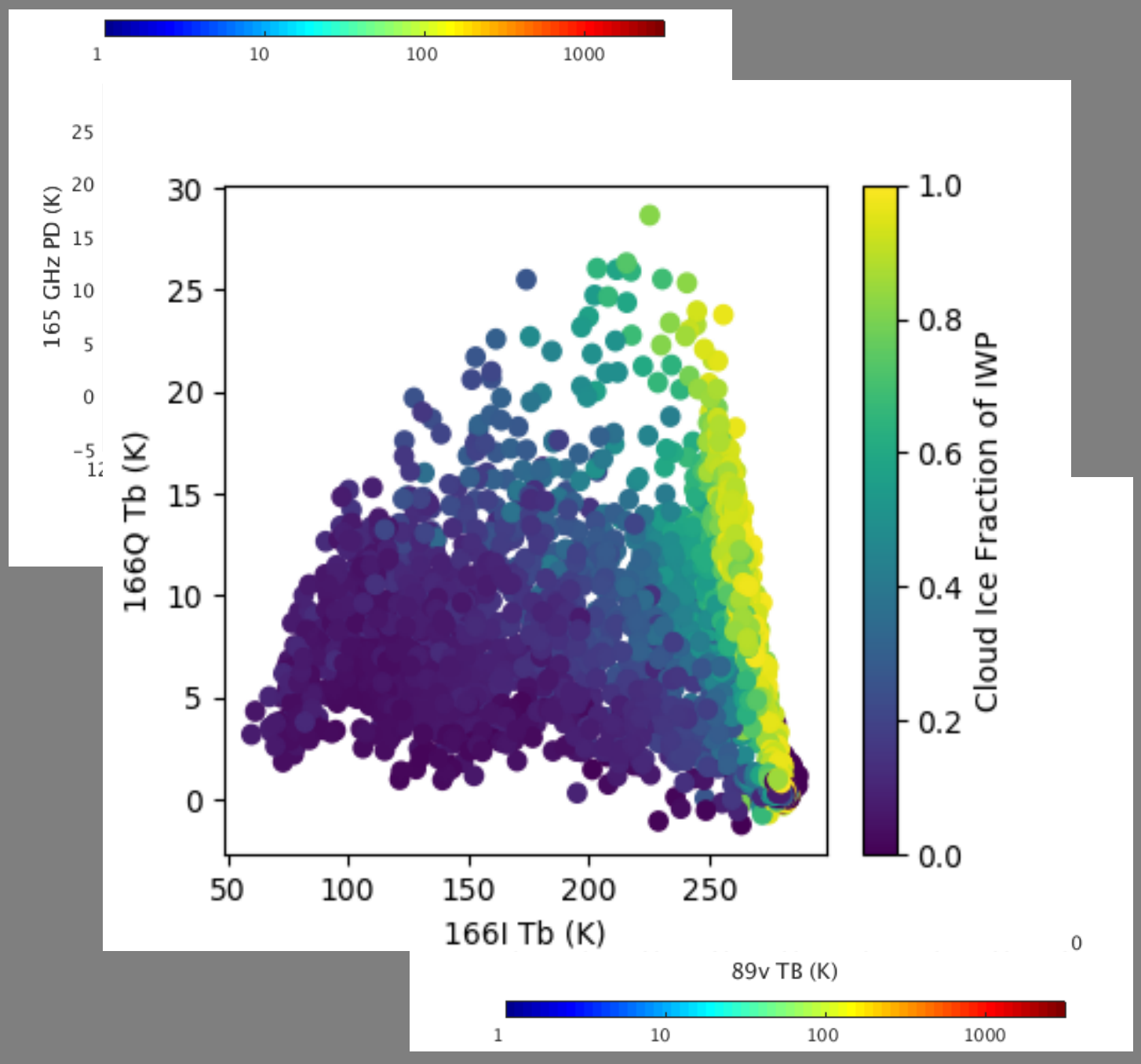




\section{CoSSIR $\mathrm{T}_{\mathrm{b}}$-W Correlations}

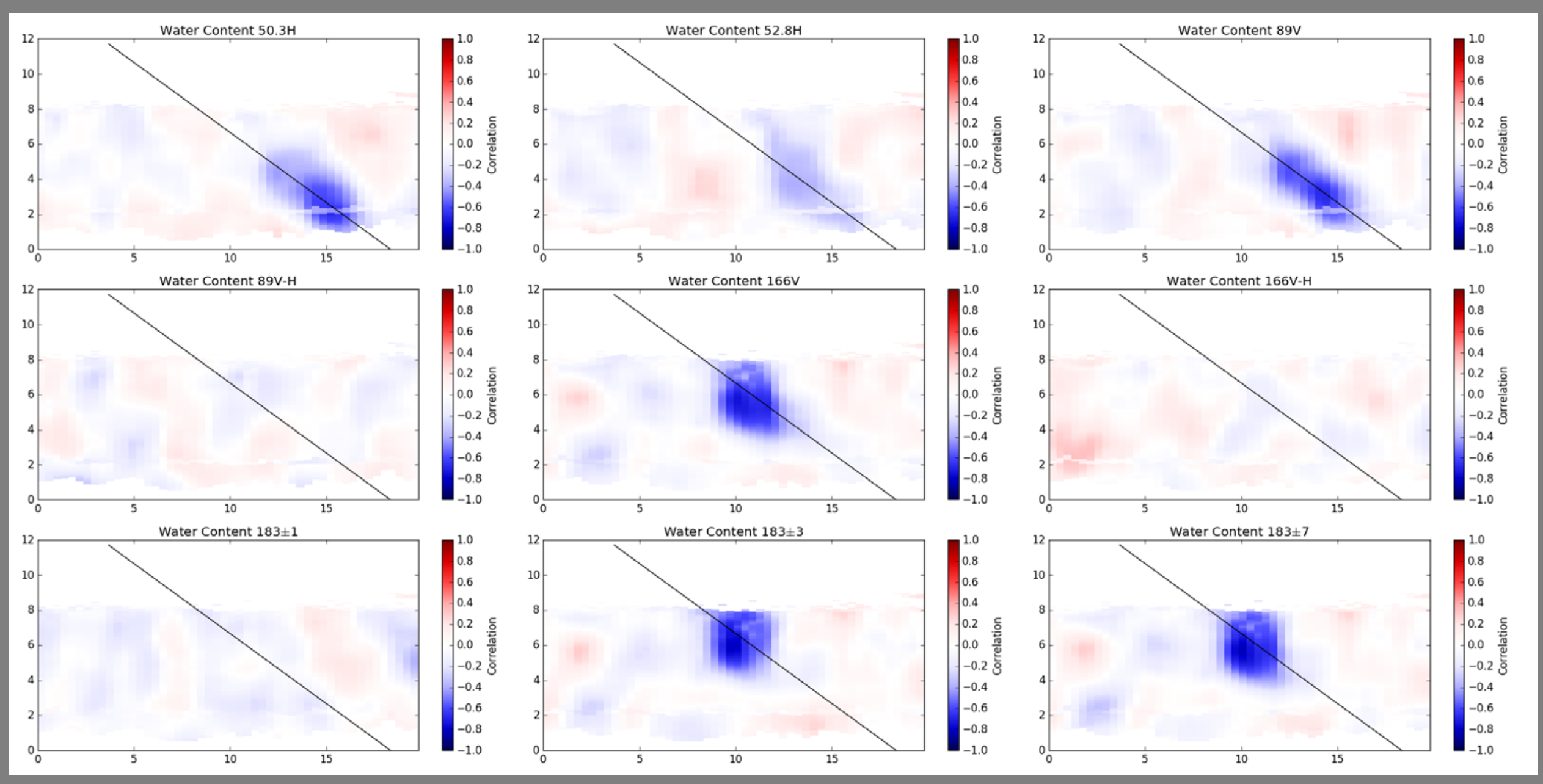




\section{Summary}

\section{Mulit-frequency radar retrievals}

- Partitioning of hydrometeor species

o Pristine

o Aggregates

o Cloud liquid

- Good qualitative agreement with in situ probe data

Additional information in polarized brightness temperatures

- Aligned versus randomly oriented particles

- Presence of supercooled liquid

o Liquid at cloud tops 


\section{Future work}

- Other OLYMPEX cases

o Interesting microphysics

- Riming

- Polycrystals

- Multi-platform observations o CRS/HIWRAP on ER-2

- Melting particles

- Aligned ice

o Scattering using IITM

- Other field campaigns

o MC3E

o IPHEX
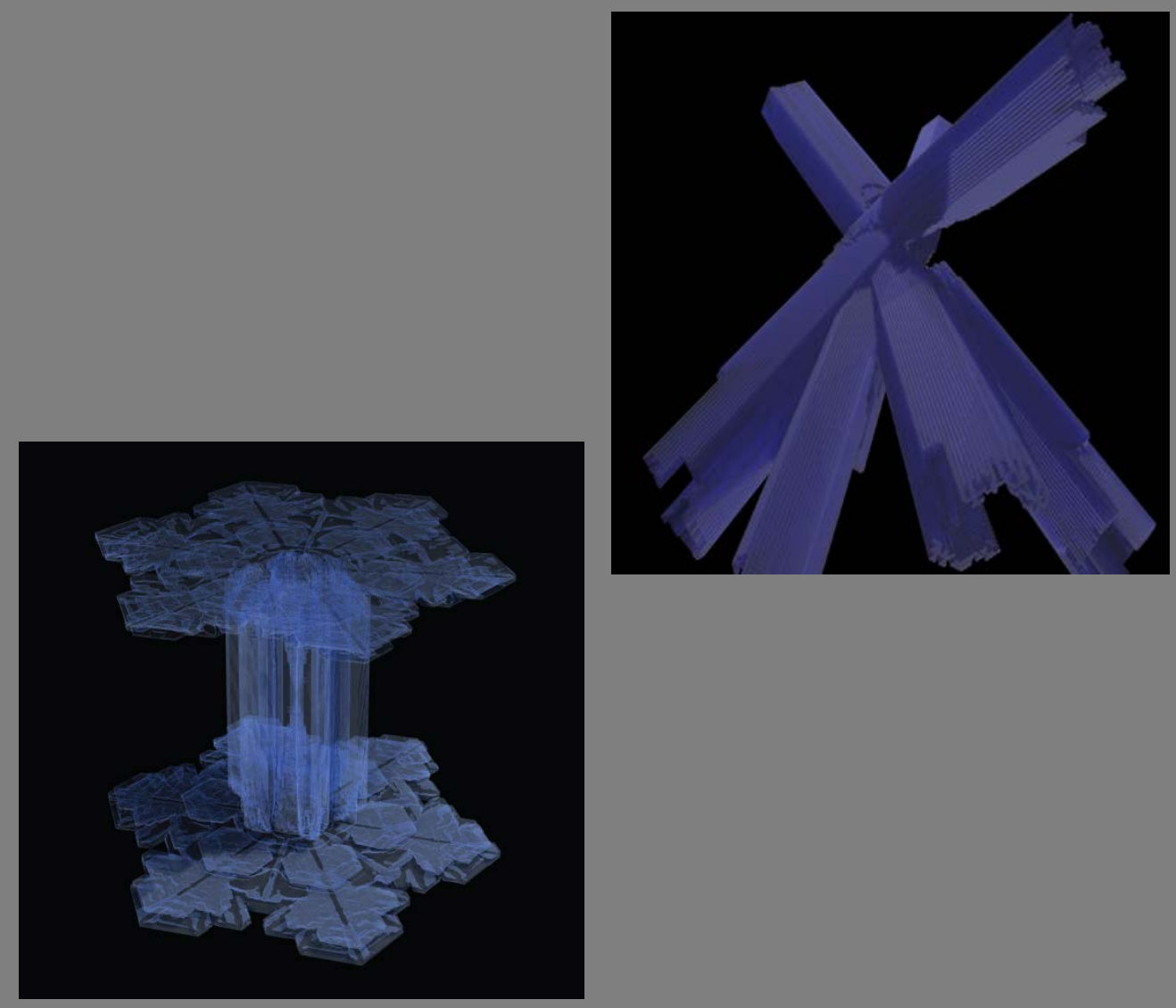